\title{
Pandemia y protesta social en América Latina: tendencias, actores y demandas de la conflictividad social y laboral en Brasil, Argentina, Chile y Colombia. 2019-2020
}

\section{Agustín Nava y Juan Grigera}

Consejo Nacional de Investigaciones Científicas

y Técnicas. Universidad Nacional de Quilmes

agustinnava82@hotmail.com

ORCID: 0000-0002-5191-2777
Department of International Development,

King's College London

juan.grigera@kcl.ac.uk

ORCID: 0000-0001-6254-7907

Title: Pandemic and Social Protest in Latin America: Trends, Actors and Demands of Social and Labor Conflict in Brazil, Argentina, Chile and Colombia. 2019-2020.

Resumen: En este artículo presentaremos un mapa de la protesta social en Brasil, Argentina, Chile y Colombia durante el periodo 2019-2020, exponiendo desde un abordaje cuantitativo las tendencias, intensidades, formatos, actores y demandas de la conflictividad social y laboral. El objetivo es, en primer lugar, pensar en términos comparativos cuáles son los cambios y continuidades de la conflictividad social y laboral en el escenario post-pandemia. Por otro lado, buscamos también dar cuenta de cuáles son las tendencias comunes y disimilitudes de la protesta social en cada uno de los países de América Latina seleccionados. Palabras clave: protesta social - conflictividad laboral - pandemia - América Latina

DOI: https://doi.org/10.46688/ahmoi.n20.347

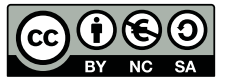

Obra bajo licencia Creative Commons 4.0 International (Atribución - NoComercial - Compartirlgual) 


\begin{abstract}
This article maps social protest in Brazil, Argentina, Chile and Colombia during the 2019-2020 period, exposing, from a quantitative approach, the trends, intensity, formats, actors and demands of social and labor conflict. The purpose is to analyze the relationship between social protest and pandemics from a broad time frame (2019-2020) and in comparative terms. The objective is to think about the changes and continuities of social and labor conflict in the post-pandemic scenario. In addition, it also seeks to account for the common trends and dissimilarities of social protest in the selected cases.
\end{abstract}

Keywords: social protest - labor conflict - pandemic - Latin American

Recepción: 18 de octubre de 2021. Aceptación: 30 de noviembre de 2021 .

$$
* * *
$$

\title{
Introducción
}

Si la pandemia de covid-19 ha puesto en agenda la discusión del conflicto social a nivel global, provocando la intervención de analistas inesperados de este terreno, ${ }^{1}$ en América Latina ya desde 2019 las protestas en varios países de la región como Chile, Ecuador o Colombia la habian puesto en primera plana. En este artículo, buscamos establecer un balance del impacto de la pandemia sobre la protesta social en la región.

La pandemia permitió teorizar sobre el impacto que las restricciones impuestas como medidas "sanitarias" tendrían sobre el volumen, la dinámica y la intensidad del conflicto social. Por un lado, en tanto las medidas estaban destinadas a restringir la movilidad de las personas y/o prohibir el uso de espacios públicos, siendo acompañadas además de la expansión del poder de policía a distintas fuerzas de seguridad, se podia suponer que esto iba a tener el efecto de refrenar las protestas o al menos redireccionarlas hacia formas que no implicaran el uso del espacio público. Este supuesto llevó incluso a especular sobre las motivaciones detrás de estas restricciones, en un amplio espectro de objeciones razonables a los alcances de la legislación de emergencia (ver por ejemplo para Europa Civil Liberties Union Europe 2021 o el debate en torno a la foto de Piñera en Plaza Dignidad/Baquedano) hasta la contrafáctica negación de las bases epidemiológicas de la pandemia.

Por otra parte, un número de factores apuntaban, por el contrario, a un sustancial incremento de la conflictividad social: en primer lugar, la profundidad de la reestructuración en curso y la peculiaridad de la "hibernación" de la actividad productiva con su desigual impacto han

1. Es el caso, por ejemplo, del Fondo Monetario Internacional o de la ciencia política mainstream que no suelen hacerse eco de este tema. 
incrementado los motivos de disconformidad y malestar no solo del movimiento obrero, sino también de otros sectores sociales. En segundo término, vinculado a esto, la pandemia evidenció el lugar esencial en la producción de algunos sectores y trabajadorxs (por ejemplo, en el caso de la salud, pero también logística o IT, la cadena de abastecimiento de alimentos, etc.), lo que podría derivar en un fortalecimiento de su poder de negociación. También inciden en el mismo sentido, la crisis de legitimidad de los Estados ante las limitaciones evidenciadas en responder a la pandemia (tocando un vértice sensible de sus ficcionales "funciones esenciales", el del garante del bien común) y la profundidad de las crisis económicas como consecuencia de los programas de rescate. Por añadidura, este shock impacta sobre un conjunto de fragilidades estructurales que son comunes a los países latinoamericanos y que solo es posible nombrar aquí: un escenario internacional de crisis, décadas de precarización del empleo, crecimiento de la desigualdad, estancamiento del crecimiento económico, mayor endeudamiento, etc. En América Latina la pandemia está golpeando a mercados laborales caracterizados por altos niveles de informalidad, sostenidas tasas de desocupación e importantes niveles de subocupación (CEPAL, 2019a).

En suma, una lectura apriorista del conflicto podria resumirse en estas preguntas: ¿es posible protestar bajo una pandemia cuando existen reglas y razones para el aislamiento? Pero también, ¿'es posible no protestar durante la pandemia cuando esta profundizó una crisis de dimensiones inéditas? A su vez, ¿cómo se han visto transformadas las formas de la protesta? Este conjunto de preguntas fundamentales que nos permiten abordar la dinámica del conflicto social contemporáneo, y también anticipar los caminos posibles (e imposibles) en la evolución de esta crisis, son también preguntas dificiles de responder sin un abordaje empírico sobre los patrones y dinámicas efectivas de la protesta social. La indagación teórica nos señala las tendencias y contratendenias en juego, pero no el resultado efectivo de su interacción.

En este trabajo, entonces, presentaremos un mapa de la protesta social en Brasil, Argentina, Chile y Colombia durante el periodo 20192020, exponiendo algunos resultados de una investigación en curso que registra y analiza, desde un abordaje cuantitativo, las tendencias, intensidades, formatos, actores y demandas de la conflictividad social. Hemos seleccionado estos cuatro casos en la medida en que son las cuatro mayores economías de América del Sur.

Presentaremos en particular los resultados del procesamiento secundario de la base de datos ACLED (que registra protestas y manifestaciones callejeras de un amplio arco de sujetos sociales). ${ }^{2}$ La relación

2. La investigación está desarrollada en el marco del proyecto "Social unrest in times 
que media entre la pandemia y la protesta social ha comenzado a dar lugar a una diversa bibliografia, ya sea desde un enfoque cuantitativo o cualitativo (Bloem y Salemi, 2021; Metternich, 2020; Murillo, 2021; Barrett y Chen, 2021; Natalucci et al., 2020; SISMOS, 2020). Sin embargo, la mayoría de los trabajos que abordan un análisis empírico de la conflictividad se centran en los casos nacionales sin establecer un marco comparativo a nivel regional y además en general abordan un espacio temporal acotado. Dentro de los pocos estudios comparativos sobre conflictividad social en el contexto de pandemia, llevados a cabo desde el punto de vista cuantitativo, podemos citar el informe ACLED (2020) sobre los casos de Brasil y Nicaragua durante los primeros meses de la pandemia y los de Bloem y Salemi (2021). Más allá de cierto acuerdo respecto a que la pandemia no supuso un impedimento para el desarrollo de la protesta social, de todos modos, no hay una profundización sobre cuáles son los cambios y continuidades en lo que respecta a sus características.

En este sentido, la finalidad de este artículo es abordar la relación entre protesta social y pandemia desde un marco temporal de veinticuatro meses (enero de 2019 a diciembre de 2020) y comparativo. En particular, nos proponemos dos objetivos. En primer lugar, pensar cuáles son los cambios y continuidades de la conflictividad social y laboral en el escenario post-pandemia: ¿La protesta social se intensifica o se atenúa? ¿Se registran cambios en sus modalidades y sujetos o su morfología no se modifica? Por el otro lado, buscamos también dar cuenta de cuáles son las tendencias comunes y disimilitudes de la protesta social en cada uno de los países de América Latina seleccionados, ya sea en términos generales o con respecto a la irrupción de la pandemia: ¿Quiénes son los sujetos que mayor predominancia presentan en el plano de la protesta? ¿Cuáles son sus principales demandas y modalidades de acción? Como veremos a continuación, más allá del impacto en un primer momento y de diferencias específicas de cada uno de los países, la conflictividad social no se suspende en el contexto de la pandemia. Este patrón es consistente con lo señalado a nivel global (Bloem y Salemi, 2021; Gerbaudo, 2020). Sin embargo, nuestra perspectiva nos permite

of covid-19" financiado por King's College London y dirigido por Juan Grigera, del que forman parte Agustín Nava (Universidad Nacional de Quilmes y Conicet), Luciana Zorzoli (Cardiff University) y Aswin Chansrichawla (King's College London). Para este artículo se utilizaron los datos para Argentina, Brasil, Chile y Colombia, tanto los provenientes de la metodologia Global Social Protest (Johns Hopkins University) como ACLED reclasificados a los fines de estudiar protesta social. ACLED ("Armed Conflict Location \& Event Data Project") es un proyecto de recopilación, análisis y mapeo de eventos de protesta y violencia politica registrados en distintas regiones del mundo, con particular énfasis en violencia armada. 
identificar las modificaciones que se producen al interior del campo de la protesta social.

El artículo se organiza en 4 secciones y las conclusiones. Inicialmente, presentaremos los aspectos metodológicos de la investigación desarrollada. En las dos secciones siguientes, analizaremos las tendencias generales de la conflictividad social y los sujetos que motorizan la misma. En el cuarto apartado nos detendremos específicamente en las dinámicas de la conflictividad laboral. Finalmente, en las conclusiones, recuperamos las principales ideas desarrolladas a lo largo del texto.

\section{Notas teórico-metodológicas}

Como ya sostuvimos, nuestro objetivo es establecer un mapa de la conflictividad social y laboral desde un abordaje cuantitativo. Las investigaciones cuantitativas sobre conflictividad social son un ejercicio que presenta cierta complejidad y no está exento de dificultades y limitaciones teóricas y metodológicas, al tiempo que es pasible de ser llevado a cabo de diversas maneras (Franzosi, 2017; Stiberman, 2017). En nuestro caso hemos decidido seguir un enfoque similar al método de Protest Event Analysis (Hutter, 2014) que consiste básicamente en registrar una secuencia temporal de acciones conflictivas llevadas a cabo por distintos sujetos sociales en relaciones con otros actores o instituciones sociales, que con distintos niveles organizativos buscan la consecución de una o más demandas, defender situaciones existentes, concretar determinados intereses o promover proyectos sociales y políticos alternativos. Para ello, la base de datos abierta de ACLED resulta de inestimable valor. La misma está construida en base a un registro y codificación de acciones de violencia política y protesta, llevada a cabo por varixs investigadorxs (que además incluye tres instancias de revisión para asegurar validez, precisión y relevancia) en base al relevamiento de una multiplicidad de portales de noticias específicos de cada país. ${ }^{3}$ Además, como se utilizan las mismas reglas respecto a las variables quién, qué, dónde y cuándo, permite llevar a cabo análisis comparativos con una alta rigurosidad. ${ }^{4}$

3. Que además suelen cubrir un criterio regional, evitando así la sobrerrepresentación de algunas regiones (en particular las capitales y centros urbanos más importantes) como consecuencia de la elección de determinados diarios o periódicos.

4. En cada uno de los países se suelen publicar series históricas de alcance nacional tanto de conflictividad laboral y social. Sin embargo, los diversos criterios teóricos y metodológicos, el alto nivel de agregación de los datos presentados y cierta dilación en su publicación no nos permite llevar a cabo el ejercicio comparativo que intentamos llevar a cabo en este trabajo. 
Nuestro uso de la base ACLED incluye un procesamiento secundario de la misma, pues la base de datos clasifica una multiplicidad de eventos de protesta y violencia política, pero incluye a su vez un desglose y detalle de cada evento. En nuestro análisis hemos incluido seis variables centrales de las acciones de protesta en función del método escogido: dónde, cuándo, quién, contra quién, cómo y por qué. Utilizando esta descripción hemos enriquecido la base a los fines de nuestro análisis, incluyendo una tipificación simplificada de los sujetos, una tipificación de las demandas (que ACLED solo incluye como parte de la descripción), de los sectores en disputa y de si los eventos sucedieron antes o después de las medidas de aislamiento en cada país.

Una segunda dimensión específica de nuestra utilización de ACLED es la selección de acciones de conflictividad social y laboral y la exclusión de otros eventos registrados que responden a fenómenos de seguridad ciudadana o seguridad pública, tales como linchamientos a delincuentes comunes, secuestros violentos, acciones armadas de organizaciones militares y paramilitares, represiones policiales, atentados de grupos del crimen organizado entre otras. En este punto cabe una aclaración. ACLED registra fundamentalmente acciones en donde el conflicto se exterioriza a través de alguna manifestación material pública, tales como manifestaciones callejeras, actos y concentraciones, tomas de establecimientos, cortes de rutas, etc. Claramente éstas son solo una de las diversas formas en las que se expresa la protesta social (que incluyen desde las manifestaciones verbales y escritas hasta los paros o huelgas, pasando por las asambleas, entrevistas, paritarias, etc). Sin embargo, distintos estudios señalan la pertinencia de utilizar este repertorio de acción como indicador de las tendencias de la conflictividad social y laboral.

Por añadidura, en el contexto de la pandemia el foco en la protesta en el espacio público se vuelve particularmente relevante a la luz de las hipótesis antes mencionadas: las medidas de aislamiento y distancia social podrian haber afectado particularmente esta forma de expresión del conflicto social. Cabe una salvedad a las restricciones de esta aproximación. Es plausible asumir que algunos sectores pueden haber tenido como estrategia la utilización de otros canales de protesta. Hemos explorado esta opción de modo limitado y no encontramos sustento para abordarla de modo sistemático. Además, este es siempre el caso con el estudio de la conflictividad: la relación entre conflicto y protesta no es lineal.

Teniendo en cuenta estas definiciones y aclaraciones sobre la selección, nuestra base de datos cuenta con 6.472 unidades de análisis para el caso de Brasil, 4.073 para Argentina, 1.614 para Colombia y 4.454 en Chile, para los veinticuatro meses que van de enero de 2019 
a diciembre de 2020. Comparando estos resultados con otras fuentes de datos nacionales hemos encontrado que los resultados son fiables. En el caso de Argentina la base resultante arroja un promedio diario de 5,5 conflictos. Dicho promedio se ubica en el mismo rango, aunque levemente inferior, al que registra con una metodologia similar el estudio de Natalucci et al. (2020) para el periodo que va entre el 16 de marzo y el 7 de junio de $2020(6,1)$. La salvedad sin embargo es que dicho trabajo utiliza una unidad de análisis más amplia que la nuestra, ya que incluye manifestaciones escritas, huelgas y paros, etc. Además, hay una coincidencia en lo que respecta a la distribución regional de la protesta: mientras que en el estudio de Natalucci et al. (2020) el 68,2\% de las protestas se desarrollan en el interior del país y el 31,7\% en el AMBA (Área Metropolitana de Buenos Aires), en nuestro caso los valores son $72,6 \%$ y $27,4 \%$ respectivamente. En el caso de Chile, el informe anual del Observatorio de Conflictos del Centro de Estudios de Conflicto y Cohesión Social-COES (2020) registra para el segundo semestre de 2019 2.700 eventos de protesta. Mientras que en nuestro caso tenemos 2.198. De manera que si calculamos el promedio diario nos da nuevamente un valor que se ubica en el mismo rango: 14,7 para el informe del COES y 12 en nuestro caso. Para Brasil, contamos únicamente con el registro de huelgas de DIEESE. Aun cuando esta es una forma específica de protesta y de un sujeto particular, una comparación de las tendencias de huelgas relevadas por DIEESE para el año 2020 muestra una evolución mensual similar (ver Huelgas por mes, DIEESE 2021:27) y de similar composición (un predominio de los reclamos que DIEESE clasifica como defensivos). Lo anterior, en nuestra opinión, da cuenta de la representatividad de nuestra muestra. Sin embargo, es necesario recordar que la finalidad de este tipo de análisis no es establecer la cantidad de conflictos, sino poder determinar con cierta rigurosidad las distintas pautas o tendencias que adquiere la conflictividad social y laboral.

\section{Tendencias generales}

El primer hallazgo de esta aproximación es que los países bajo estudio muestran altos niveles de conflictividad general, al menos desde un punto de vista cuantitativo. Los promedios mensuales de acciones de protesta para el periodo 2019-2020 son: Brasil (270), Argentina (170), Chile (185), Colombia (67). Sin embargo, si tenemos en cuenta los distintos tamaños poblaciones, los países en donde la intensidad de la protesta es mayor son Chile y Argentina con una tasa de incidencia ${ }^{5}$

5. Tasa de incidencia de acciones de protesta [Eventos de protesta/población total $\mathrm{x}$ 100.000]. Los datos de población los hemos extraído de CEPAL (2019b). 
de 23,4 y 9,09 conflictos cada 100.000 habitantes, respectivamente, en comparación con tasas de incidencia de 3,06 y 3,02 en Brasil y Colombia, respectivamente. ${ }^{6}$

En segundo lugar, es posible diferenciar dinámicas nacionales particulares dentro de un ciclo general común signado por la pandemia. Hay que tener en cuenta que en los casos analizados, los gobiernos a partir del mes de marzo de 2020 han adoptado 5 tipos de medidas para contener la propagación de los contagios, que resultaron altamente disruptivas para el normal desenvolvimiento de la actividad económica y social (Filgueira et al., 2020): cierre de escuelas y centros educativos, confinamientos en el hogar (voluntarios u obligatorios), restricción de la presencialidad de actividades en las empresas consideradas no esenciales, prohibición de eventos públicos y de realización de viajes y traslados al interior del país. De todos modos, en cada uno de estos países las modalidades e intensidades de estas restricciones han sido disimiles. Cómo se puede ver en el Cuadro 1, podríamos ubicar a Brasil como el caso en donde la profundidad de estas medidas ha sido menor, a Chile en una posición intermedia y a Colombia junto a Argentina como los casos en donde las medidas tuvieron mayor intensidad, por lo menos durante gran parte del año $2020 .{ }^{7}$

Tal como puede apreciarse en el Gráfico 1, más allá de estas diferencias, todos los países muestran una caída substancial del conflicto abierto en los meses que siguen a la irrupción de la pandemia y al inicio de las restricciones, y también un rápido crecimiento de la cantidad de

6. Resulta sugerente el hecho de que los países seleccionados que registran niveles comparativamente bajos de conflictividad social y laboral (Brasil y Colombia) son los que al mismo tiempo están más afectados por acciones armadas y conflictos violentos que hemos excluido de nuestra muestra (véase más arriba). Si consideramos la totalidad de acciones registradas en la base de datos ACLED, este último tipo de hechos representa el 62\% de las acciones en Brasil y el 42,4\% en Colombia. Por el contrario, en Argentina y Chile solo significan una pequeña parte del registro: $1,5 \%$ y $4,2 \%$ respectivamente.

7. Con cierto desfasaje temporal, también los países fueron implementando variadas medidas sociales y económicas para mitigar y contener los efectos adversos de las restricciones. Sistematizarlas en su totalidad es de suma complejidad, en parte porque las acciones gubernamentales se modifican en periodos cortos de tiempo. Sin embargo, como se desprende del relevamiento realizado por Filgueira et al. (2020), en primer lugar, en los cuatro países se han fortalecido esquemas existentes (y establecido nuevos) de transferencias monetarias e implementado subsidios estatales a los ingresos laborales. En materia de empleo, surgen algunas diferencias. Por ejemplo, solo en el caso de Argentina se estableció la prohibición de los despidos, mientras que en Chile y Colombia no se implementaron licencias especiales por covid-19, como en Brasil y Argentina. 
conflictos en los tres meses siguientes. Sin embargo, veremos que esto se sobreimpone a dinámicas específicas en cada uno de los países.

En el caso de Argentina, se puede identificar un ciclo de alza sostenido cuyo inicio coincide con las elecciones presidenciales en agosto de 2019. Luego se aprecia una brusca interrupción en el mes siguiente a la irrupción de la pandemia. Finalmente, el tercer ciclo se abre en mayo de 2020 cuando la conflictividad no solo retoma sus niveles previos a la pandemia, sino que además los supera ampliamente, alcanzando el pico de todo el periodo en septiembre de 2020 con 453 acciones mensuales.

En los casos de Chile y Brasil la dinámica de la protesta está marcada por picos de conflictividad durante determinados meses que superan ampliamente los promedios mensuales. Como se puede observar en

\section{Cuadro 1. Profundidad de las restricciones*. Brasil, Chile, Argentina y Colombia. (1 de marzo - 20 de agosto de 2020)}

\begin{tabular}{|l|c|c|c|c|c|}
\hline & Trabajo & Colegios & E. Públicos & Viajes & Confinamiento \\
\hline Brasil & 39 & 85 & 0 & 42 & 24 \\
\hline Chile & 49 & 86 & 40 & 42 & 45 \\
\hline Argentina & 52 & 85 & 43 & 84 & 52 \\
\hline Colombia & 63 & 90 & 45 & 83 & 50 \\
\hline
\end{tabular}

* 100 equivale a restricción total.

Fuente: elaboración propia en base a Coronavirus Government Response Tracker, Blavantik School of Public Policy, U. Oxford.

Gráfico 1. Conflictividad social.

Brasil, Argentina, Chile y Colombia, 2019-2020

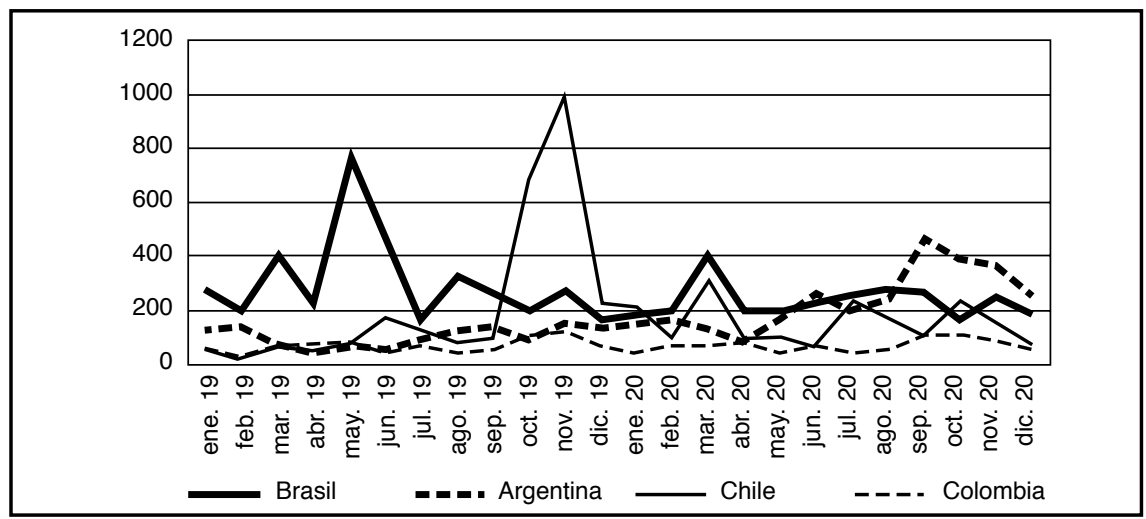

Fuente: Social Unrest in Times of Covid, procesamiento secundario de ACLED 
el Gráfico 1, la conflictividad social en Chile presenta una tendencia bastante lineal durante la primera mitad del 2019, que es interrumpida abruptamente por lo que se ha denominado el "estallido social". Es decir, las jornadas de masivas y radicalizadas protestas de octubre y noviembre de dicho año iniciadas el 18 de octubre luego de que se anunciara un alza programada del pasaje del metro en Santiago de Chile. Estas alcanzaron picos de 615 y 1.005 eventos por mes, que quintuplican el promedio mensual del período. De todos modos, luego de las jornadas de octubre y noviembre la conflictividad cae abruptamente y retoma su tendencia algo lineal, a pesar de que durante el mes de marzo de 2020 la protesta se intensifica levemente como consecuencia de las diversas acciones exigiendo medidas al inicio de la pandemia, por las manifestaciones del movimiento de mujeres en el Día Internacional de la Mujer y por las manifestaciones en apoyo al plebiscito para modificar la Constitución. A su vez, las protestas exigiendo una reforma constitucional que permita a los afiliados a las Aseguradoras de Fondos de Pensiones (AFP) retirar hasta el $10 \%$ de sus ahorros de la jubilación para enfrentar la crisis económica, van a marcar un nuevo pico de conflictividad durante el mes de julio de 2020.

En el caso de Brasil la tendencia de la conflictividad presenta una línea en forma de serrucho siendo más intensa en los primeros meses de 2019 , cuyo pico es el mes de mayo de dicho año con casi 800 acciones, motorizadas por las masivas protestas contra el recorte al presupuesto para educación impuesto por el gobierno del presidente Jair Bolsonaro y en rechazo a la propuesta de reforma jubilatoria. Las protestas contra estas iniciativas del gobierno nacional continúan durante los meses subsiguientes (incluyendo la realización de una importante huelga general el 14 de junio). Sin embargo, hacia fines de 2019 la conflictividad empieza a perder intensidad. A partir de la pandemia la protesta presenta una leve tendencia hacia arriba (alcanzándose un pico durante el primer mes de la misma), aunque en niveles menores a los de 2019.

Por su parte, en Colombia la tendencia de la conflictividad es más fluctuante, alcanzando el pico de la conflictividad, al igual que Chile, durante los meses de octubre y noviembre de 2019, con 101 y 124 acciones de protesta respectivamente. Impulsadas fundamentalmente por las movilizaciones convocadas por docentes, estudiantes y centrales obreras contra las iniciativas del gobierno nacional de llevar a cabo reformas laborales, pensionales y recortes en el presupuesto educativo. En particular, los manifestantes exigen politicas para superar las tasas nacionales de desempleo, la desigualdad económica y la violencia contra los activistas sociales. En este caso, la caída no es tan abrupta luego del momento más álgido. A su vez, a diferencia de la Argentina, los primeros dos meses de la pandemia no parecen modificar la tendencia 
al alza de la conflictividad que se inicia en enero de 2020, aunque sí en los meses subsiguientes, retomando el ciclo de alza hacia fines de año.

En términos generales, la incidencia de la pandemia en la dinámica de la conflictividad parece circunscribirse a la reducción del número de conflictos durante los primeros dos meses de la pandemia. Por fuera de estos, la tendencia general retorna a su dinámica previa, sin que se verifique ni una caída sostenida ni un aumento de los conflictos donde estos no venían en aumento. En los casos de Chile y Brasil, el momento más álgido de la protesta es durante el año 2019, mientras que en Argentina las acciones de protesta se intensifican en el escenario post-pandemia (el promedio mensual de acciones pre y post-pandemia es de 109,7 y 253,7 respectivamente). En Colombia la intensidad de la protesta pre y post-pandemia no se modifica sustancialmente (el promedio mensual de acciones es de 64,3 en los meses previos a la pandemia y de 70,3 con posterioridad a la misma).

Si bien la pandemia en términos generales no ha significado una interrupción generalizada y sostenida de la protesta, la misma sí ha debido adaptarse a las condiciones impuestas por el nuevo escenario. A pesar de que las tendencias cuantitativas de la protesta no se modifican en gran medida luego de la irrupción del covid-19, de todos modos se producen cambios en lo que respecta a la fisonomía de la misma si tenemos en cuenta los actores y las demandas de los conflictos.

\section{Sujetos de la protesta}

Hemos encontrado en este estudio dos aspectos para destacar sobre los sujetos de la protesta social. ${ }^{8}$ En primer lugar, se destaca la alta presencia de acciones de protesta protagonizadas por sujetos que no presentan ninguna afiliación institucional o corporativa (descriptos en las noticias como "vecinos", "ciudadanos", o sencillamente "manifestantes", etc.), que en nuestra codificación aparecen con la sigla SIIC (sin identificación institucional o corporativa). Este sector, en verdad, es altamente heterogéneo en su interior y con una multiplicidad de demandas, aunque se pueden reconocer dos tipos de situaciones: por un lado, acciones en donde en general los diversos y heterogéneos sujetos

8. Cabe aclarar que las acciones en las que intervienen varias personificaciones sociales hemos decidido atribuírselas, en primer momento, al sujeto principal o al que tiene la iniciativa, ya que en los cuatro casos considerados se dan múltiples combinaciones que si se repusieran en su totalidad dificultarian el análisis y sobredimensionarian el peso de algunos sujetos. Si bien nuestra recodificación nos permitiria en un segundo momento ponderar qué porcentaje de acciones realiza el sujeto principal con otras personificaciones sociales, por razones de espacio no lo presentaremos en esta oportunidad. 
no se encuentran institucionalizados y se activan contra situaciones particulares o para resolver problemas concretos; por otro lado, como veremos en particular en el caso chileno, incluye eventos de protestas en donde la categoría de manifestantes subsume una diversidad de identidades corporativas previamente movilizadas.

En segundo lugar, el movimiento obrero es el sujeto con pertenencia corporativa o institucional que mayor activismo y presencia tiene en el campo de la protesta social, aunque no en la misma proporción y dinámica en cada uno de los países. Esta tendencia responde probablemente a factores estructurales y coyunturales: el mayor "poder estructural" y "asociativo" del movimiento obrero, por un lado, y por otro que en el contexto de la crisis post-pandemia se ha intensificado la tensión capital y trabajo.

La predominancia del movimiento obrero es claramente mayor en el caso argentino. Como se puede apreciar en el Grafico 2a, en promedio el $41 \%$ de las acciones de protesta se corresponden a conflictos laborales, promedio que sin embargo se reduce levemente en el momento post-pandemia (40\%). De todos modos, gran parte del crecimiento de la protesta en los primeros meses post-pandemia se explican por las acciones del movimiento obrero. Esto resulta particularmente significativo si tenemos en cuenta que básicamente estamos haciendo mención a manifestaciones o movilizaciones en los lugares de trabajo o en la vía pública, acciones que a priori se verian obstaculizadas en el contexto de aislamiento físico.

\section{Gráfico 2a. Sujetos de la conflictividad social. Argentina, 2019-2020}

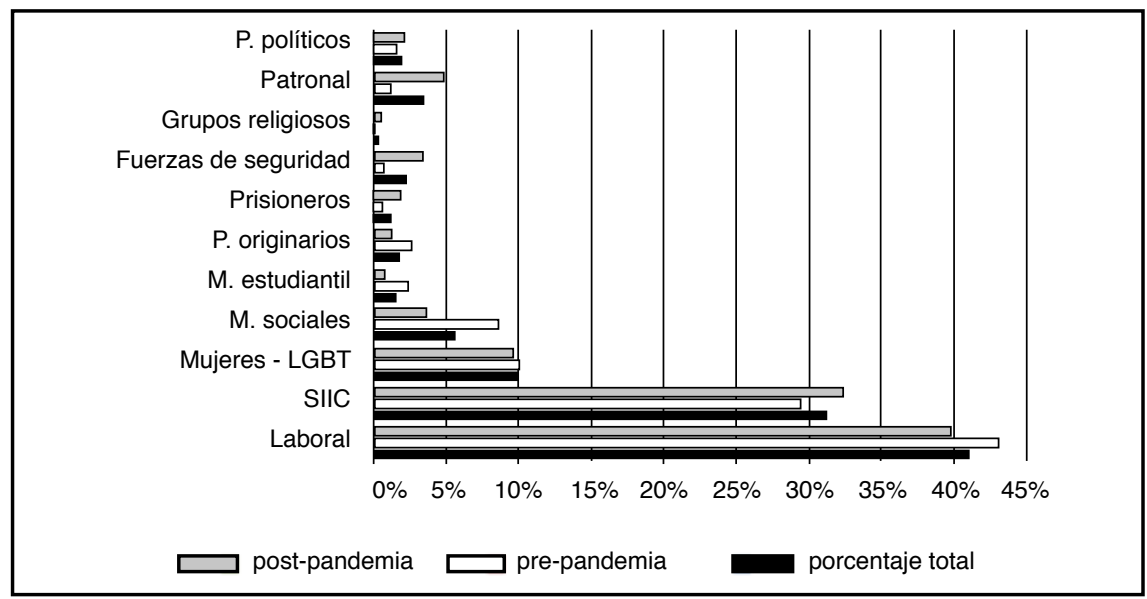

Fuente: Social Unrest in Times of Covid, procesamiento secundario de ACLED. 
En orden de importancia lo siguen los movimientos de mujeres y LGBT $(10 \%)$ y otras organizaciones sociales $(5 \%)$, aunque en el primer caso mantienen estable su participación mientras que en el último caen fuertemente en importancia después de la pandemia, ya que pasan de representar el 8,7\% de las acciones en el momento previo al 3,6\% posteriormente. En el caso argentino, las estrictas medidas de aislamiento debilitaron la habitual y extraordinaria capacidad de movilización que tienen los movimientos sociales, debiendo canalizar sus demandas por otros medios (Ferrero y Natalucci, 2020). En el caso del movimiento de mujeres, la particularidad es que los eventos del calendario (en febrero demandas por la legalización del aborto, el dia internacional de la mujer el 8 de marzo, en junio Ni Una Menos, en noviembre las marchas por el dia internacional contra la violencia hacia la mujer) marcan una parte cuantitativa importante de la movilización. Es de destacar que luego de declarada la pandemia una parte importante de las manifestaciones están vinculadas a denuncias de feminicidios y otras formas de violencia de género y doméstica que se incrementaron sustancialmente en el marco del aislamiento social. ${ }^{9}$

El movimiento estudiantil, por su parte, es el único que efectivamente deja de tener presencia como consecuencia de la pandemia, aunque ya mostraba una conflictividad bastante reducida en el periodo anterior (1,4\% del total de conflictos). Aspecto que se contrapone a lo que sucede en los otros países considerados, como veremos más adelante.

En el caso argentino, tres sectores son los que aumentan su participación en el campo de la protesta en el escenario post-pandemia: por un lado, los sectores patronales $(1,4 \%$ a $6 \%$ respectivamente), motorizados fundamentalmente contra las iniciativas del gobierno nacional con respecto al sector agropecuario; por el otro, los prisioneros $(0,5 \%$ a $4 \%$ respectivamente), protagonistas de un ciclo de protestas en el inicio de la pandemia solicitando liberaciones y otras medidas de prevención. Y finalmente la categoria SIIC $(29,5 \%$ a $32,4 \%$ respectivamente), que alcanza su pico en el mes de noviembre con casi 150 acciones de protesta, lideradas fundamentalmente por vecinos y comerciantes contra las medidas de aislamiento social y exigiendo la reapertura de las actividades económicas. La activación de estos tres sectores (sobre todo del primero y del tercero) da cuenta de la recurrencia que presenta la acción colectiva conservadora de matriz liberal (Bonavena y Millán, 2020).

Es importante señalar, en este sentido, que en el caso argentino la mayor predominancia numérica de la conflictividad laboral no parece

9. Distintos estudios (Ivandic et al., 2021; Taub, 2020) han señalado a la violencia patriarcal, como una dimensión importante durante la pandemia en una definición amplia de "conflictividad social". 
indicar en modo alguno una capacidad de articular el conflicto: tanto el análisis de las demandas como de la limitada articulación de las luchas son contundentes en este sentido, como veremos en la sección siguiente.

El caso chileno muestra patrones en cierto sentido opuestos a los de Argentina: si bien el movimiento obrero sigue siendo el actor con representación corporativa que tiene mayor presencia en la conflictividad social ( $20 \%$ de las acciones), dentro de los países analizados es el que presenta menos relevancia. Además, como se puede observar en el Gráfico 2b, el conflicto laboral no registra grandes fluctuaciones en el escenario post-pandemia. Por otro lado, en Chile el movimiento estudiantil adquiere una mayor preeminencia ubicándose en el segundo lugar con el 10\% de las acciones (nuevamente, en términos de nuestra muestra este es el caso en que tiene mayor protagonismo). Aunque aquí sí la pandemia afecta la dinámica de la protesta, ya que el movimiento estudiantil reduce fuertemente su participación en el campo de la conflictividad (del 13\% al 5\%).

Gráfico 2b. Sujetos de la conflictividad social. Chile, 2019-2020

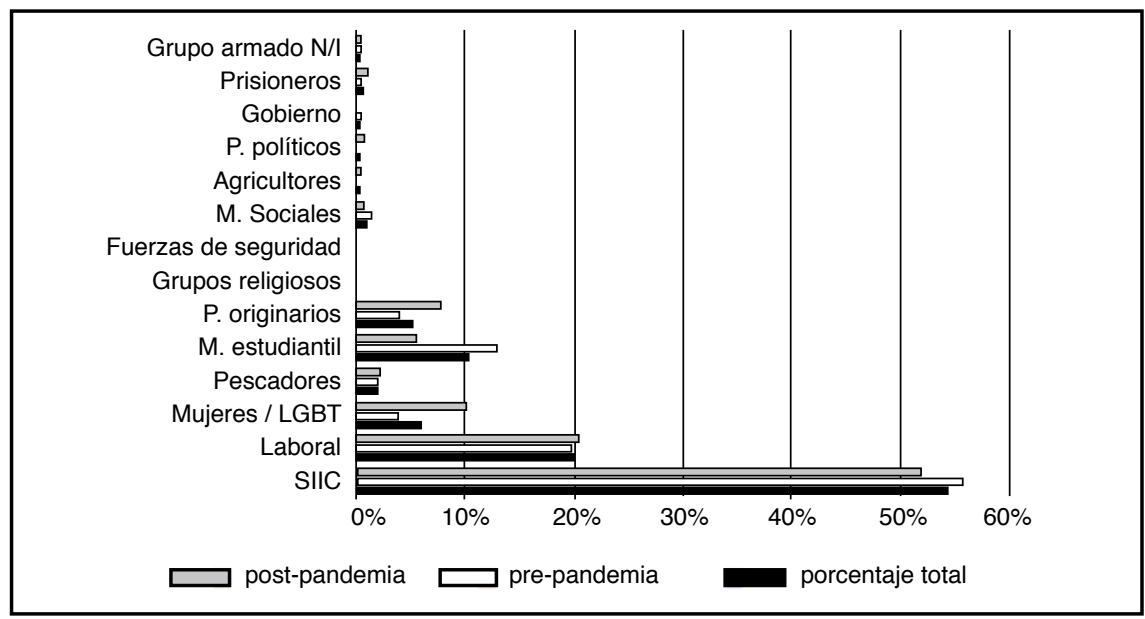

Fuente: Social Unrest in Times of Covid, procesamiento secundario de ACLED.

Sin embargo, lo que caracteriza al caso chileno es que más de la mitad del total de las acciones (54\%) responden a la categoría SIIC. Esta alta incidencia de actores no institucionalizados o corporativos es en este caso indicador inconfundible de un nivel alto de radicalización de la protesta y de amplia participación por encima de las estructuras de 
representación. Gran parte de las acciones durante las jornadas de octubre y noviembre en 2019 están protagonizados por sujetos a los cuales no se les puede adscribir una pertenencia corporativa. En este punto, todas las identidades previas (obreros, estudiantes, mujeres, pueblos originarios, movimientos sociales, etc.) se subsumen bajo la categoría de "manifestantes" en momentos donde la protesta no solo adquiere altos niveles de violencia material, sino que además involucra a amplios sectores sociales en una lucha frontal contra el régimen político y social.

Por último, en el caso chileno solo dos sectores aumentan su participación en el campo de la protesta en el escenario post-pandemia: por un lado, el movimiento de mujeres / LGBT (del 3,7\% al 10\%), aunque no por motivos estrictamente ligados con la pandemia, ya que gran parte de las acciones durante 2020 se concentran durante el dia internacional contra la violencia hacia la mujer (8 de marzo) y durante el mes de julio en rechazo a un fallo judicial que dictaba la prisión preventiva de un hombre acusado de delitos sexuales contra varias mujeres; y, por el otro, los pueblos originarios que pasan del $4 \%$ al $8 \%$, motorizados fundamentalmente por las movilizaciones del pueblo mapuche reclamando la libertad a los presos políticos pertenecientes a esa comunidad.

Por su parte, como se puede observar en los Gráficos 2c y 2d, la distribución de los sujetos en conflicto en Brasil y Colombia se asemeja a la de Chile: la preponderancia de la categoria SIIC (41\% y 39\% en Brasil y Colombia respectivamente), en segundo orden de importancia la conflictividad laboral (33\% en Brasil y 30\% en Colombia), y un protagonismo más marcado del movimiento estudiantil (8\% y $10 \%$ respectivamente) que en Argentina.

En Brasil, tanto la conflictividad laboral como la que entra dentro de la categoría SIIC se incrementan en el escenario post-pandemia (del $31 \%$ al $37 \%$ en el primer caso y del $37 \%$ al $47 \%$ en el segundo). En el segundo caso las acciones se concentran en el primer mes de la pandemia (marzo de 2020) impulsadas por dos tipos de protestas: por un lado, en un comienzo se registran varias manifestaciones de ciudadanos en general que rechazan la actitud del presidente Bolsonaro de minimizar la gravedad de la pandemia de coronavirus. Sin embargo, y a medida que algunos estados provinciales comienzan a tomar medidas de aislamiento y confinamiento como formas de prevención, empiezan a crecer los reclamos de diversos sectores exigiendo la reapertura de algunas actividades. Acciones y reclamos que continúan a lo largo de todo el año 2020.

A diferencia de estos dos sujetos, el movimiento estudiantil no mantiene los altos niveles de movilización previos a la pandemia. El movimiento estudiantil universitario brasileño inicia un ciclo de fuertes luchas, fundamentalmente durante el mes de mayo de 2019, realizadas junto 
Gráfico 2c. Sujetos de la conflictividad social. Brasil, 2019-2020

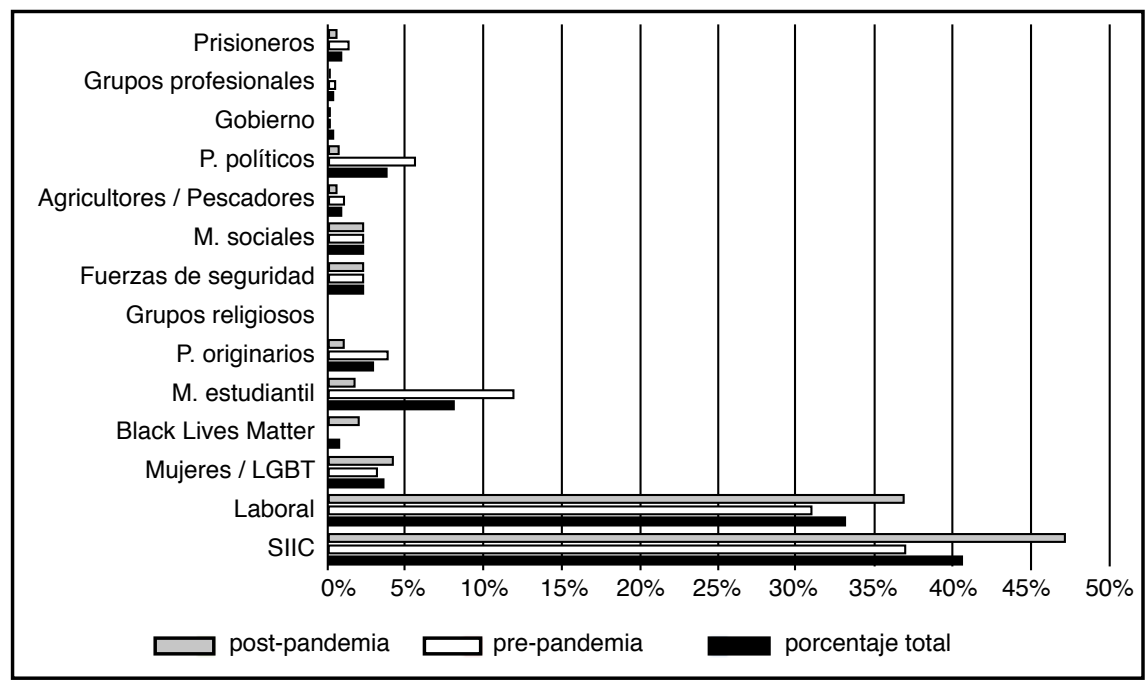

Fuente: Social Unrest in Times of Covid, procesamiento secundario de ACLED.

Gráfico 2d. Sujetos de la conflictividad social. Colombia, 2019-2020

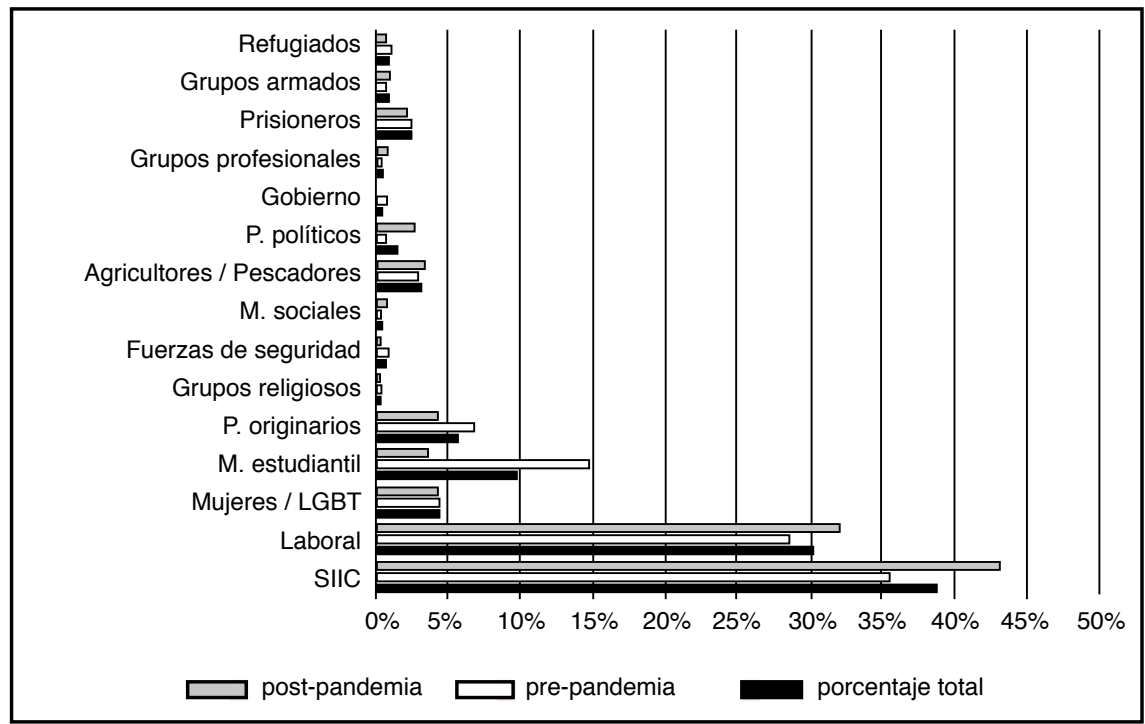

Fuente: Social Unrest in Times of Covid, procesamiento secundario de ACLED. 
a otros sectores del movimiento obrero (en particular docentes), contra los recortes en el presupuesto de educación, en defensa de la autonomía universitaria y en rechazo al proyecto "Future-se", que pretendía tercerizar el financiamiento de la educación pública. De todos modos, como en los demás países, las medidas de aislamiento y el dictado de las clases bajo la modalidad virtual representan un punto de inflexión para el movimiento estudiantil.

También en Colombia el conflicto laboral y el protagonizado por sujetos sin pertenencia institucional o corporativa adquieren una mayor centralidad con posterioridad a la pandemia (el conflicto laboral pasa del $29 \%$ al $32 \%$ y SIIC de $35 \%$ a $43 \%$ ). En este país el crecimiento de la categoría SIIC está impulsado en los primeros meses por el ciclo de protestas que exige recursos al gobierno nacional luego de que se implementara una cuarentena obligatoria; y posteriormente, por una serie de radicalizadas y masivas protestas de la población en general en contra de la violencia policial luego de que un abogado fuera asesinado por agentes policiales en el mes de septiembre, entre otros reclamos.

En este sentido, la dinámica de la protesta en Colombia en términos generales se asemeja más al caso chileno, diferenciándose de Argentina y Brasil donde el predominio de este tipo de sujeto en el campo de la protesta da cuenta más de una tendencia al crecimiento de la acción colectiva liberal-conservadora. Más aun, en términos generales, los niveles de radicalización de la conflictividad (medidos en términos del grado de violencia material presente en las acciones) son mayores en los casos de Chile, fundamentalmente, y en Colombia. En Chile, sólo el 51\% de la conflictividad social se desarrolla de manera pacífica (es decir en donde no se ejerce ningún tipo de violencia), mientras que en Colombia ese valor es del $77 \%$. Para los casos de Argentina y Brasil los valores son aún más altos: $93 \%$ y $86 \%$ respectivamente. La particularidad aquí es que, a diferencia de lo que sucede en otras latitudes (Gerbaudo, 2020), la pandemia no ha supuesto una fase de intensificación de la radicalización de la protesta, sino que por el contrario las formas de protesta pacífica se han incrementado levemente en el escenario post-pandemia: $6 \%$ en el caso de Chile, 3\% en Argentina, 4\% en Brasil y 14\% en Colombia.

\section{Conflictos laborales}

¿Cuáles son los cambios que pueden registrarse en la conflictividad laboral? Si bien, a excepción de Argentina, los conflictos clasificados como SIIC son más significativos, el análisis de la composición y demandas del sujeto corporativo de mayor peso reviste un interés en sí mismo.

En primer lugar, debe señalarse que el análisis de la composición de los sectores y las demandas que articulan los conflictos muestra un 
cambio substancial antes y después de la pandemia. A su vez, en los cuatros países los sectores y las demandas que crecen en intensidad y las que disminuyen son casi las mismas (aunque el peso de cada una de ellas al interior de cada país sea diferente, ver Gráficos 3 (a, b, c y d) y 4 (a, b, c y d) ). En particular, crecen los sectores más afectados directamente por la pandemia y las medidas de aislamiento, y las demandas que presentan un carácter más "defensivo".

El análisis de los sectores muestra que en el escenario post-pandemia hay tres que incrementan su presencia en el campo de la protesta: salud, servicios y comercio y transporte y logística. En algún punto, ésta es una tendencia previsible en la medida en que han sido los sectores que más han sufrido un impacto directo de la pandemia o de las medidas destinadas a controlarla, ya sea por una intensificación de su trabajo en condiciones precarias y sin las medidas e instrumental de prevención suficientes (como en el caso del personal de salud), como por el impacto directo de la contracción de la actividad económica que ha afectado particularmente a sus actividades. Por ejemplo, en lo que respecta a lxs trabajadorxs del sector comercio al comienzo de la pandemia exigen que sus actividades sean clausuradas como medida de prevención, aunque a medida que transcurre el tiempo y la crisis económica se agudiza los reclamos son por la reapertura de sus actividades, en particular en los países como Argentina que tuvieron una cuarentena extensa, o exigiendo a los gobiernos la implementación de medidas que garanticen un minimo de ingreso.

Por el otro lado, pierden importancia la administración pública (con la excepción de Colombia), educación, el sector industrial y centrales sindicales y nucleamientos gremiales. A excepción de este último, una posible explicación de la pérdida relativa de protagonismo de estos sectores en el contexto de la pandemia es una estabilidad comparativamente mayor en el empleo y en las condiciones de trabajo, en tanto son actividades en las que no se registran grandes procesos de despidos, suspensiones o rebajas salariales en comparación con los casos mencionados previamente. Con respecto a la sensible caída de las acciones de protesta motorizadas por las centrales sindicales, nucleamientos gremiales y/o alianzas entre diferentes sindicatos (que por ejemplo en el caso de Chile pasan del $25 \%$ al $2 \%$ ), esta tendencia es bastante significativa si reparamos en que en el escenario pre-pandemia la conflictividad laboral, en general, acusa cierta propensión a centralizarse en instancias de coordinación o unificación. Los conflictos protagonizados por las centrales o alianzas entre gremios destacan por su alta incidencia en el plano del conflicto, ya que representan el $44 \%$ de las acciones en Colombia, el $27 \%$ en Brasil, el 25\% en Chile y el $18 \%$ en Argentina. Entendemos que la causa de este cambio responde a dos procesos diferenciados en 
los distintos países analizados. Por un lado, en casos como Argentina o Brasil, la hipótesis que proponemos es que el contexto defensivo propio de la situación de pandemia obstaculiza los procesos de articulación de las protestas y despolitiza el conflicto. Por ejemplo, se comprueba que las luchas adquieren un carácter más localizado. En cambio, en Chile y Colombia es la radicalización del conflicto la que desplaza a los sujetos corporativos del centro de la escena.

Un análisis de las demandas muestra que en su gran mayoría estas son contra el Estado y contra sus políticas (con excepción de Argentina): antes de la pandemia estas representan el 67\% de las acciones en Chile, el $54 \%$ en Colombia, el $41 \%$ en Brasil y solo el $22 \%$ en Argentina. ${ }^{10} \mathrm{La}$ crisis como consecuencia de la pandemia marca en el conflicto laboral un punto de inflexión claro: las luchas en contra del gobierno o contra politicas del mismo se discontinúan sensiblemente en el contexto post-pandemia (del $67 \%$ al $28 \%$ de las acciones en Chile, del $41 \%$ al $10 \%$ en Brasil, del 54\% al 25\% en Colombia y del 22\% al $11 \%$ en Argentina), ${ }^{11}$ al tiempo que aumentan significativamente las acciones reclamando distintas formas de continuidad laboral ${ }^{12}$ y los reclamos salariales ${ }^{13}$ en particular exigiendo pagos adeudados o contra la reducción de los salarios. Como respuesta a la crisis, las empresas adoptaron medidas que atentaron contra la estabilidad en el empleo (despidos más suspensiones) y en los ingresos, u otras formas de vulneración como la desregulación con suspensiones y disminución salarial. En este sentido, como sostienen Natalucci et al. (2020) para el caso argentino, en el contexto de la pandemia las demandas no estuvieron dirigidas a la ampliación de derechos, sino que por el contrario estuvieron orientadas más a exigir la garantía o restitución de derechos ya adquiridos, alcanzando la protesta un carácter más sectorial.

Más allá de estas tendencias generales en común, se pueden señalar algunas heterogeneidades en cada uno de los países considerados, ya que varian considerablemente cuáles son los sectores más movilizados,

10. Cabe aclarar que, como sostiene Faundes Peñafiel (2016), el hecho de que las demandas se dirijan a las distintas instancias estatales no supone necesariamente que el objetivo último de los distintos movimientos sociales implique una lucha por el gobierno o el poder del Estado mismo.

11. En el caso de Argentina es importante considerar que hay un cambio de gobierno en diciembre de 2019.

12. Incluye acciones en defensa de las fuentes de trabajo, contra despidos y suspensiones, solicitando la apertura de nuevas fuentes de trabajo, etc. Estas pasan a ser del $18 \%$ al $23 \%$ en Argentina, del $11 \%$ al $36 \%$ en Brasil, del $6 \%$ al $11 \%$ en Chile y del $10 \%$ al $24 \%$ en Colombia.

13. Estos pasan de representar 34\% al 44\% en Argentina, del 23\% al 24\% en Brasil, del $8 \%$ al $14 \%$ en Chile y del $7 \%$ al $17 \%$ en Colombia. 
sobre todo en el momento previo a la irrupción del covid-19 (a excepción de las centrales sindicales y alianzas gremiales con cierto nivel de protagonismo en todos los países como ya sostuvimos). Por ejemplo, en el caso de la Argentina pre-pandemia (véase Gráfico 3a), la actividad de lxs trabajadorxs de la administración pública tuvo un protagonismo central en el panorama general de la conflictividad laboral (realizando 1 de cada 4 acciones, la mitad de las cuales incluían demandas salariales para enfrentar la inflación). En segundo lugar, se sitúan las luchas iniciadas por los trabajadores industriales $(13 \%)$, impulsadas en su mayoria por reclamos de continuidad laboral (59\%). Recordemos que durante el último periodo de la presidencia de Mauricio Macri un importante número de empresas cerraron plantas de producción e incluso abandonaron el país, a la par que se paralizaron varios proyectos de obras públicas.

Tanto el protagonismo de lxs trabajadorxs de la administración pública como del sector industrial en el escenario pre-pandemia es particular del caso argentino. Por el contrario, en los demás casos, si excluimos la variable "Centrales y nucleamientos", los trabajadorxs de la educación y los que pertenecen al sector transporte y logistica son los que mayor relevancia relativa tienen en el plano de la protesta laboral, aunque en distintas proporciones en cada país y por motivos en parte similares. Lxs trabajadorxs de la educación protagonizaron el 26,5\% de las acciones en Chile, el $16 \%$ en Brasil y el 20,5\% en Colombia antes de la pandemia, movilizados en general contra las políticas de los gobiernos nacionales.

Por su parte, las acciones protagonizadas por el sector transporte y logística representan el $15 \%$ en Chile, el $21 \%$ en Brasil y el $17 \%$ en Colombia. Si bien en los tres casos hay un reclamo común referido a mejorar las condiciones de trabajo y seguridad, dentro de este grupo se incluyen luchas de distinto tipo e incluso contrapuestas. En este sentido, se pueden mencionar las acciones de protesta vinculadas de algún modo con la introducción de las nuevas tecnologias digitales, ya sean los reclamos de los taxistas en contra del funcionamiento y legalización de plataformas de transporte tales como Uber, como las acciones de trabajadorxs de plataformas (tanto de transporte como de reparto) exigiendo regularizar sus actividades. En este punto deberiamos destacar que, en el contexto de la pandemia, tienen lugar varias huelgas de lxs trabajadorxs de la economía de plataforma, que presentan la particularidad de que logran coordinarse a nivel regional, abarcando varios países de América Latina (Howson et al., 2020). Es obvio que las condiciones impuestas por la pandemia no solo han dado impulso a la actividad de las plataformas sino que también han acentuado la precariedad de sus condiciones de trabajo (Gutiérrez y Atzeni, 2021). 


\section{Gráfico 3a. Sector de actividad de las acciones del movimiento obrero. Argentina, 2019-2020}

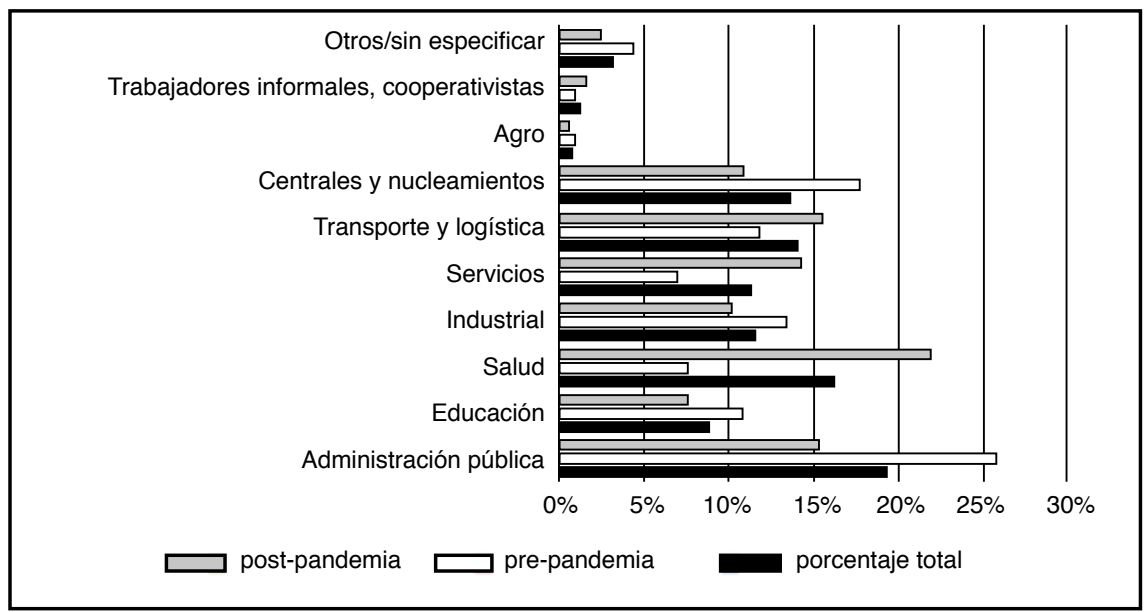

Fuente: Social Unrest in Times of Covid, procesamiento secundario de ACLED.

Gráfico 4a. Demandas de las acciones del movimiento obrero. Argentina, 2019-2020

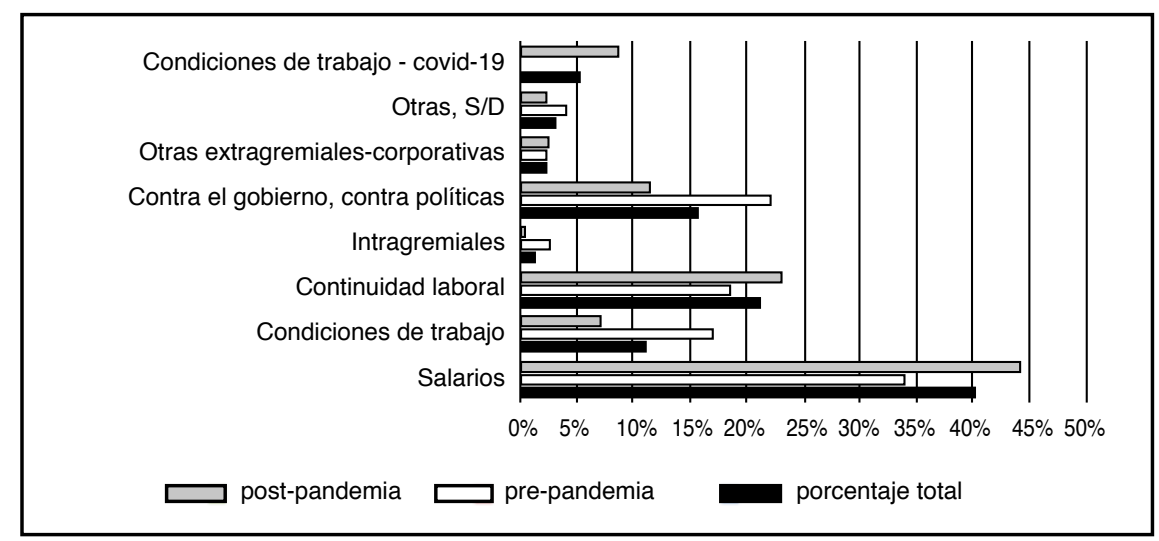

Fuente: Social Unrest in Times of Covid, procesamiento secundario de ACLED. 


\section{Gráfico 3b. Sector de actividad de las acciones del movimiento obrero.} Chile, 2019-2020

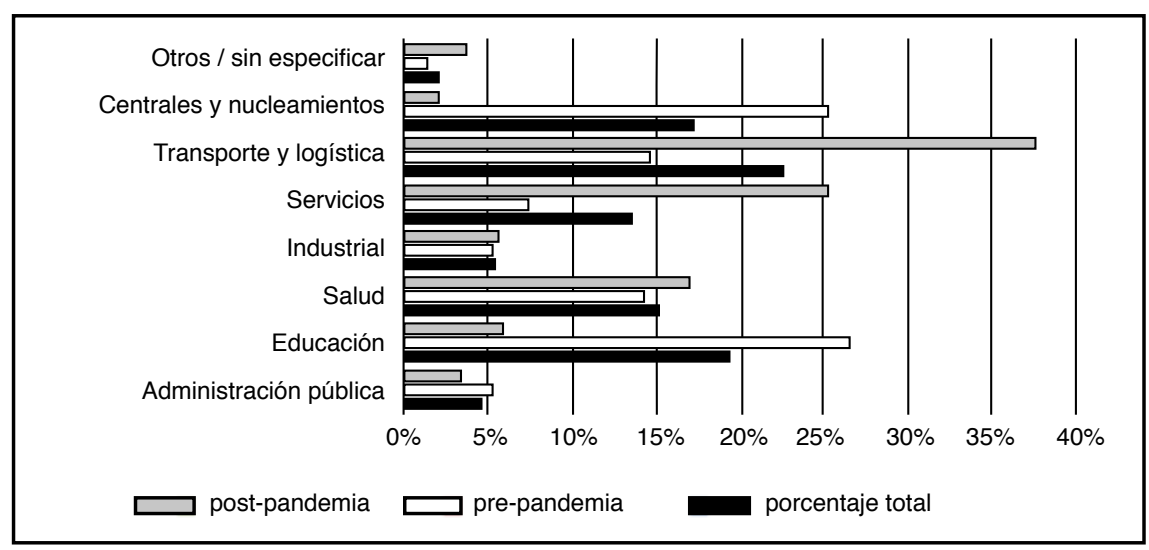

Fuente: Social Unrest in Times of Covid, procesamiento secundario de ACLED.

Gráfico 4b. Demandas de las acciones del movimiento obrero. Chile, 2019-2020

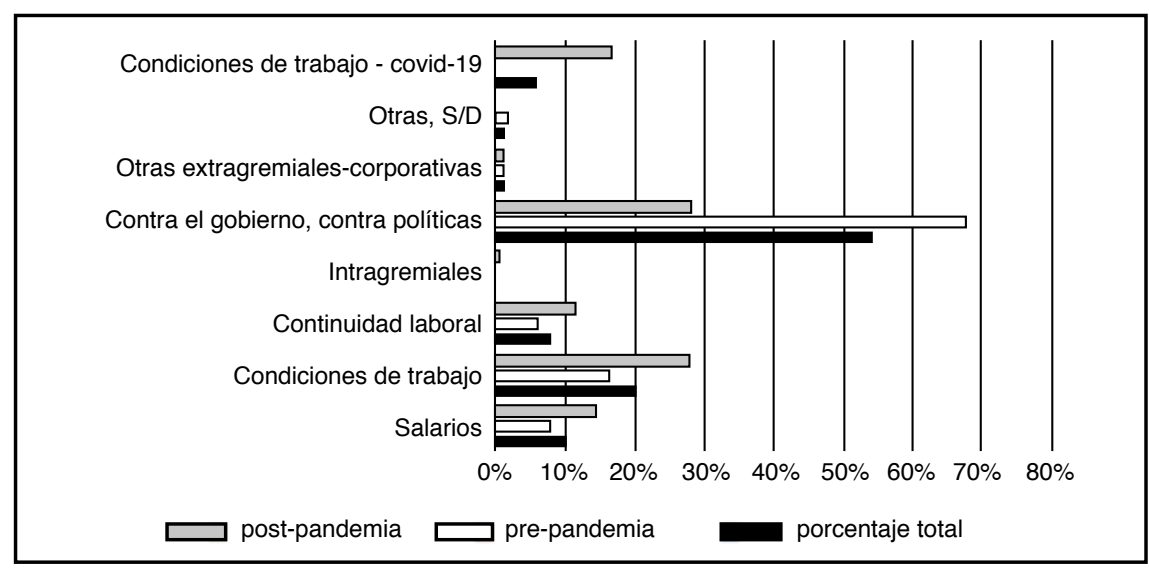

Fuente: Social Unrest in Times of Covid, procesamiento secundario de ACLED. 


\section{Gráfico 3c. Sector de actividad de las acciones del movimiento obrero. Brasil, 2019-2020}

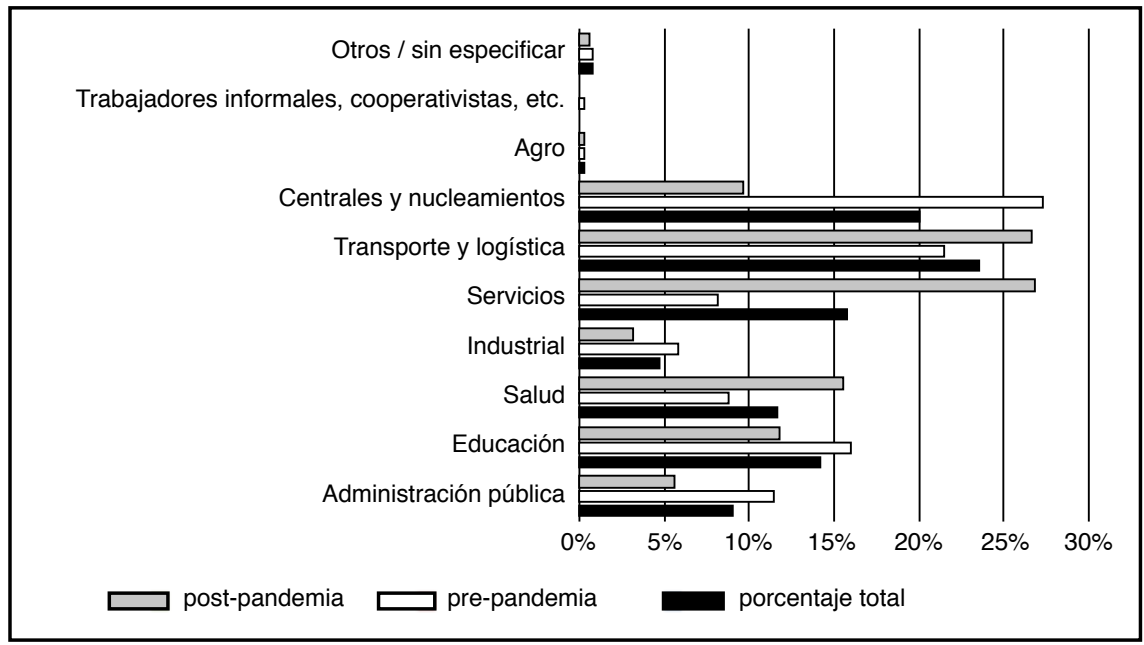

Fuente: Social Unrest in Times of Covid, procesamiento secundario de ACLED.

\section{Gráfico 4c. Demandas de las acciones del movimiento obrero. Brasil, 2019-2020}

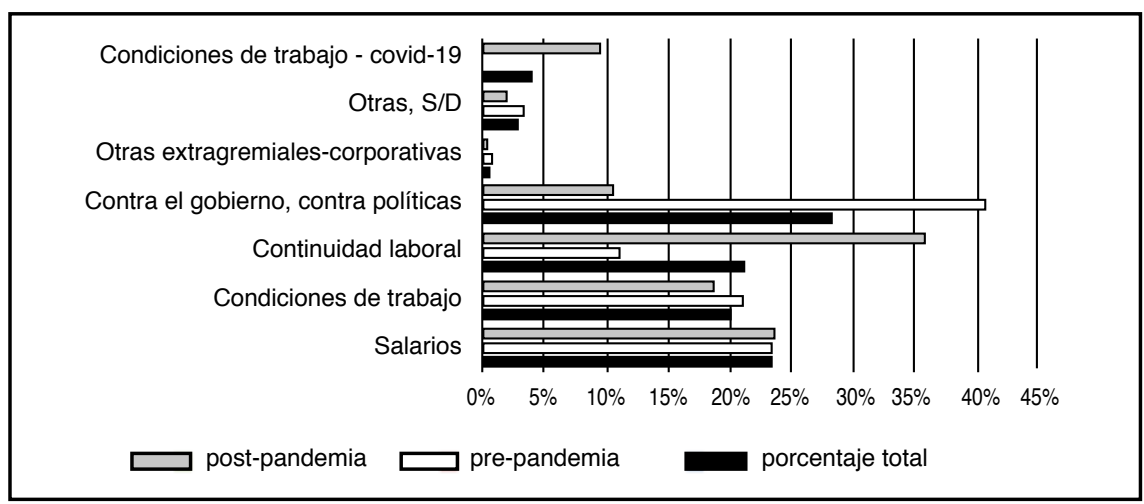

Fuente: Social Unrest in Times of Covid, procesamiento secundario de ACLED. 


\section{Gráfico 3d. Sector de actividad de las acciones del movimiento obrero.} Colombia, 2019-2020

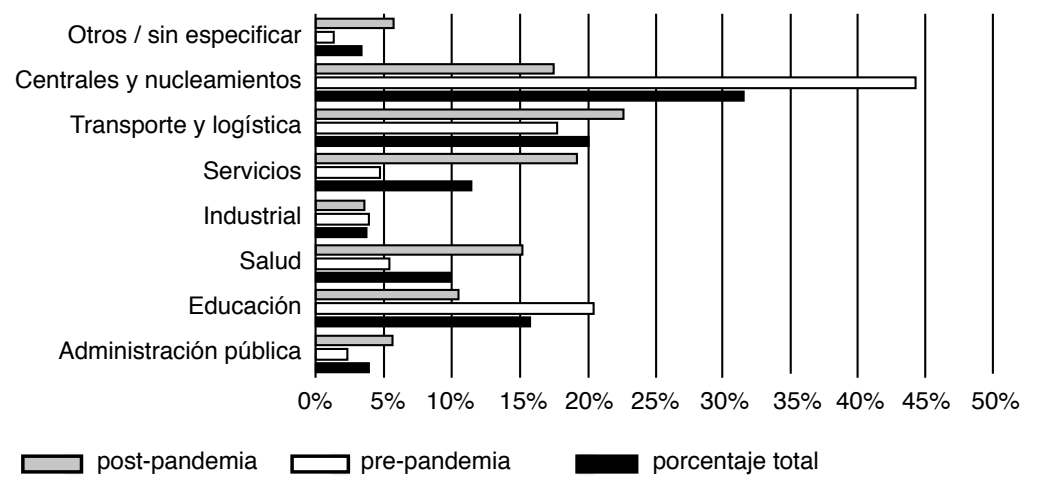

Fuente: Social Unrest in Times of Covid, procesamiento secundario de ACLED.

\section{Gráfico 4d. Demandas de las acciones del movimiento obrero.} Colombia, 2019-2020

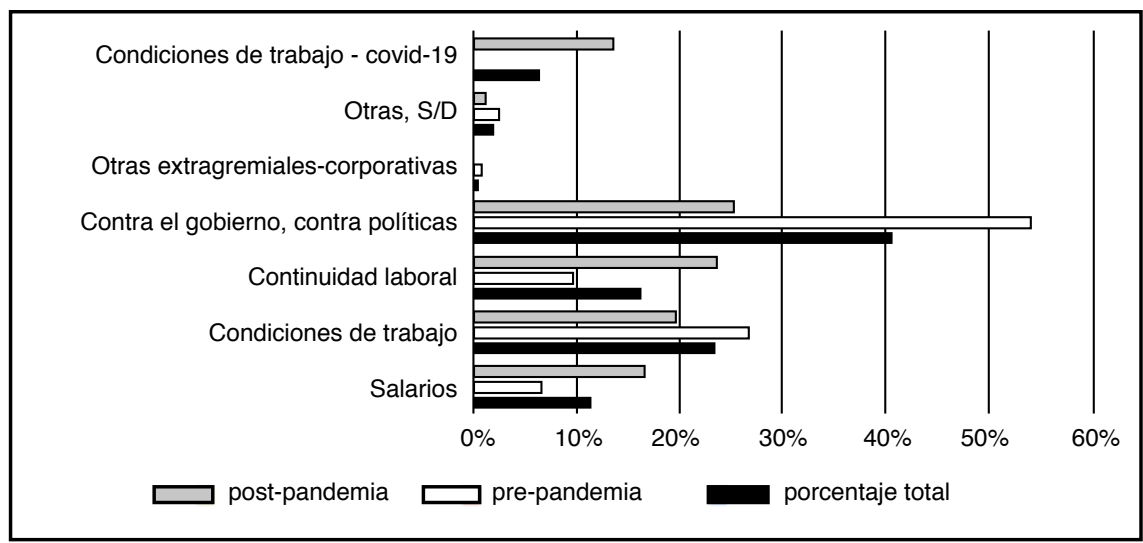

Fuente: Social Unrest in Times of Covid, procesamiento secundario de ACLED. 


\section{Conclusiones}

¿Cuáles son las conclusiones a las que este ejercicio de análisis cuantitativo de la protesta social nos permite arribar? Entendemos que, en sintesis, se puede afirmar primero que el impacto de la pandemia en la conflictividad de América Latina es limitado, y segundo que la dinámica del conflicto en múltiples dimensiones analizadas muestra un patrón diferenciado entre Chile y Colombia por un lado, y Argentina y Brasil por otro.

Al comienzo nos preguntábamos si la protesta social se había intensificado o atenuado en el escenario post-pandemia. Tal como hemos visto, en ninguno de los países estudiados la pandemia ha modificado sustancialmente las tendencias de la conflictividad. No se ha producido una disminución sostenida de la protesta más allá del breve período de uno o dos meses y si algún efecto es posible asociar a la crisis generada por la pandemia (sin que la relación de causalidad quede totalmente clara), es cierta intensificación de la protesta social (en Argentina y Colombia, por ejemplo).

Es, entonces, posible afirmar categóricamente que los obstáculos que las múltiples reglas de distanciamiento social y mayor poder de policía imponen a las capacidades de organización y exteriorización del conflicto social fueron menos importantes que las tendencias coyunturales y estructurales que animan la protesta de conjunto. La excepción a esta dinámica global la constituyen sectores específicos (algunos movimientos sociales y particularmente el movimiento estudiantil) que requeririan estudios de caso para comprender acabadamente de qué modo se traslada el impacto de la pandemia en desmovilización (¿ha sido la falta de presencialidad y su impacto en la socialización?, ¿o el desplazamiento de los reclamos a otras esferas?).

Nuestro análisis de las demandas del conflicto laboral también mostró con claridad que la fisonomía que adquiere la conflictividad conforme se desenvuelve la crisis post pandemia da cuenta de una conflictividad "defensiva", con demandas asociadas a pérdidas de derechos y protestas reactivas.

Pero si estos son los elementos que podemos sintetizar de la dinámica global de la conflictividad, el análisis también muestra que la dinámica de la conflictividad en Chile y Colombia es singular en la región y que la comparación con Argentina y Brasil no hace más que poner de manifiesto sus peculiaridades. En primer lugar, en ambos casos vemos un proceso de radicalización que se expresa en el predominio de un sujeto diverso sin identificación partidaria clara ni tampoco demandas específicas reportadas por la prensa. Es significativo señalar (como han hecho especulativamente Murillo, 2021, o Barbosa Dos Santos, 2021) 
que los "estallidos" suceden en países que no pasaron por la "Ola Rosa" o la serie de gobiernos progresistas o neopopulistas de la década pasada (Grigera, 2017; Piva, 2013; Modonesi, 2014). Entendemos que esto antes que responder a los mayores o menores niveles de redistribución debe buscarse en los mecanismos de institucionalización y desmovilización de la protesta que el ciclo neopopulista encarnó. Tal como hemos señalado en otra oportunidad, múltiples analistas de los gobiernos neopopulistas tienen un fuerte acuerdo de que "la firma de su gobierno fue, si acaso, la desmovilización" (Anderson, 2011, p. 7), o en palabras de Singer, la "pasteurización" de la movilización social (Singer, 2012, p. 21). Son estos legados del lulismo y del kirchnerismo los que operan explicando la dinámica actual de Argentina y Brasil.

En suma, el impacto global de la pandemia parece ser limitado al momento de explicar la dinámica de la conflictividad en los casos estudiados (contrario a la rápida correlación que establecen Bloem y Salemi, 2021, o Barrett y Chen, 2021). En términos de la región, la crisis que se ha desenvuelto a partir del fin del ciclo de los altos precios de los commodities y que ha sido profundizada por los efectos de la pandemia resulta tener mayor importancia (tal como muestran las temporalidades de la protesta). Un segundo elemento que resulta relevante es si los países pasaron recientemente por procesos de institucionalización del conflicto, tal como lo han hecho los países en la "ola progresista" o neopopulista (contrario a quienes han intentado explicar esto a partir de las formas institucionales siguiendo la ciencia politica mainstream). Por último, la dinámica concreta de la protesta ha respondido a características locales, de impacto global y condiciones coyunturales compartidas con la región.

Las formas y dinámicas de las protestas y "estallidos" están dando forma a los modos de "manejo" de la crisis. En tanto la crisis actual de largo alcance no ha terminado, podemos decir que el futuro todavía no llegó.

\section{Referencias}

ACLED (2020). Pandemic and Political Unrest in Brazil and Nicaragua. Armed Conflict Location \& Event Data Project. En línea https://acleddata. com/curated-data-files. Consultado en mayo de 2021.

Anderson, P. (2011). Lula's Brazil. London Review of Books. 33 (7), pp. 3-12. Barbosa Dos Santos, F.L. (2021). Rebeliones pandémicas en América del Sur. En Jacobin. https://jacobinlat.com/2021/08/16/rebeliones-pandemicas-en-america-del-sur/

Barrett, P. y Chen, S. (2021). Social Repercussions of Pandemics. IMF Working Paper. 021. 
Bloem, J.R. y Salemi, C. (2021). Covid-19 and conflicto. World Development. 140, abril. https://doi.org/10.1016/j.worlddev.2020.105294.

Bonavena, P. y Millán M. (2020). El conflicto social en la Argentina pre-pandemia. Una mirada panorámica del siglo XXI con perspectiva socio-histórica. En Robinson Salazar Pérez (ed.). América Latina después del 2020. Elaleph.com.

CEPAL (2019a). Evolución y perspectivas de la participación laboral femenina en América Latina. Coyuntura Laboral en América Latina y el Caribe. 21 (LC/TS.2019/66).

CEPAL (2019b). Estimaciones y proyecciones de población a largo plazo 1950-2100. En linea: https://www.cepal.org/es/temas/proyecciones-demograficas/estimaciones-proyecciones-poblacion-total-urbana-rural-economicamente-activa.

DIEESE (2021). Balanço das greves de 2020. En linea: https: / /www.dieese. org.br/balancodasgreves/2021/estPesq99greves2021.html.

Faundes Peñafiel, J. (2016). América Latina y conflicto social en el siglo XXI: Escenarios y debates sobre el descuajeringamiento de la relación Estado, mercado y sociedad civil. Revista Chilena de Derecho y Ciencia Política. 7 (2), mayo-agosto, pp. 103-156.

Ferrero, J.P. y Natalucci, A. (2020). COVID-19 Pandemic and Social Unrest: The Social Protest by Other Means. Reflections on the Argentinian Case. Bulletin of Latin American Research, 39 (1), pp. 67-70.

Filgueira, F. y Blofield M. (2020). COVID19 and Latin America: Social Impact, Policies and a Fiscal Case for an Emergency Social Protection Floor. CIPPEC.

Filgueira, F. et al. (2020). América Latina ante la crisis del covid-19: vulnerabilidad socioeconómica y respuesta social”. Serie Politicas Sociales, 238. CEPAL.

Franzosi, R. (2017). La prensa como fuente de datos socio-históricos: Cuestiones sobre la Metodología de recolección de datos a partir de periódicos. Revista de Estudios Maritimos y Sociales, 11, pp. 255-286.

Gerbaudo, P. (2020). The pandemic crowd: protest in the time of covid-19. Journal of International Affairs, 73 (2), pp. 61-76.

Grigera, J. (2017). Populism in Latin America: Old and New Populisms in Argentina and Brazil. International Political Science Review, 38, 4, septiembre, pp. 441-55. https://doi.org/10.1177/0192512117701510.

Gutiérrez, F. y Atzeni, M (2021). Repartidores de plataformas: víctimas invisibles de la pandemia. En Observatorio Económico UAH.

Howson, K., Ustek-Spilda, F. et al. (2020). “Just because you don't see your boss, doesn't mean you don't have a boss": covid-19 and Gig Worker Strikes across Latin America. International Union Rights, 27 (3), pp. 20-28. Hutter, S. (2014). Protest event analysis and its offspring. En Della Porta (ed.), Methodological Practices in Social Movement Research. Oxford University Press, pp. 335-367.

Ivandic, R., Kirchmaier, T. y Linton, B. (2021). The Role of Exposure in 
Domestic Abuse Victimization: Evidence from the covid-19 lockdown. SSRN Scholarly Paper. Social Science Research Network. https://doi. org/10.2139/ssrn.3686873.

Modonesi, M. (2014). Conflictividad sociopolitica e inicio del fin de la hegemonía progresista en América Latina. Anuario del Conflicto Social.

Metternich, N. (2020). Drawback before the wave?: Protest decline during the covid-19 Pandemic. Disponible en: file:// C:/Users/User/AppData/ Local/Temp/protestNote.pdf.

Murillo, M.V. (2021). Protestas, descontento y democracia en América Latina. Revista Nueva Sociedad. 294. Disponible en: https://static.nuso. org/media/articles/downloads/COY_Murillo_294.pdf.

Natalucci, A., Fernández Mouján, L., Kelmeszes, A., Mate, E., Ramirez Andrade, I., Ríos, V., Stefanetti, C., y Vaccari, S. (2020). La protesta en cuarentena. Análisis de una base cuantitativa sobre protestas sociales en el marco del proyecto monitor laboral. CITRA.

Piva, A. (2013). ¿Cuánto hay de nuevo y cuánto de populismo en el neopopulismo? Trabajo y Sociedad. 21, pp. 135-157.

Singer, A. (2012). Os Sentidos do Lulismo. Companhia das Letras.

SISMOS (2020). Conflictividad social en contexto de aislamiento. En linea: https://observatoriodeconflictividad.org/conflictividad-social-en-contextos-de-aislamiento.

Stiberman, L. (2017). Conflictividad laboral en Argentina: Una aproximación a las metodologías del Ministerio de Trabajo de la Nación y la Central de Trabajadores Argentinos. Argumentos, 19, pp. 238-273.

Taub, A. (2020). A New Covid-19 Crisis: Domestic Abuse Rises Worldwide. The New York Times, 6 de abril. https:/ / www.nytimes.com/2020/04/06/ world/coronavirus-domestic-violence.html. 